\title{
Probability Field in Chaotic Intra-Entropic Interactions
}

\author{
Moshe Szweizer and Rivka Schlagbaum \\ CBRE Group Inc., Global Research and Consulting, Auckland, New Zealand \\ (Dated: February 27, 2021) - doi.org/10.31219/osf.io/y5v6d
}

\begin{abstract}
In the article, it is shown that the concept of mass can be arrived at through a consideration of two probability fields interacting with each other. The interaction is subject to discontinuities. These, in turn, when being traversed, pose a resistance, which is perceived as mass. Thus, mass is a manifestation of discontinuity in the probability field.

The approach allows for the retrieval of masses of elementary particles, providing high agreement with the experimental data. It also explains the longevity of the proton and explains why other heavy particles are short-lived. Moreover, the model presented in the paper sheds light on the nature of weak interactions.
\end{abstract}

\section{Introduction}

The paper introduces a concept of a probability field, which is arrived at within the framework of classical physics. It is important to stress that the discussion presented in this paper does not relate to any notions introduced through quantum mechanics. Rather, the probability field introduced here is derived in the context of the Boltzmann-Gibbs entropy and is employed through an interaction between two classical probability distributions. Chaos theory is used to model such interaction. The model is applied in the low probability context which allows for the development of a mechanism describing quark mass formation.

Investigation of entropic forces constitutes one of the most exciting research topics. This is partly because of the evidence of entropy influencing a wide spectrum of reality, but also due to entropy exerting such influence at the most fundamental levels. In physics, it is expected that entropy provides an agent which is expressed through the four observable forces of nature [1]. Moreover, entropy has been recognized as a major factor in information transmission theory $[2,3]$.

The paper assumes the validity of some of the currently established entropy theories. In particular, the entropic time is associated with entropy production [4,5,6], consequently, the universe evolution is associated with entropy production [7]. Entropy is assumed to constitute a fundamental force underlying physically observable reality [8].

The paper looks at a set of two feedback formulae that are connected through the standard conditional probability relationship. Such setup models an intra-entropic interaction. The attractor obtained in this way is analyzed in the context of the attractor's basin of attraction. This latter approach allows for the introduction of quantization of attractor trajectories and the introduction of the uncertainty principle.

A method of association of quark masses with probability transitions is proposed. The transitions are observed at particularly low values of entropic time, thus allowing to perceive quark formation in the context of the universe emergence.

In the second part of the paper, the model is employed to assess the masses of composite particles. In particular, the masses of the proton, neutron, sigma and $\mathrm{W}$ boson are calculated.

The paper is closed with a discussion of findings.

\section{Materials and Methods}

In the case of a two-parameter sequence, for an initial point $\left(\mathrm{p}_{0}, \mathrm{q}_{0}\right)$ the sequence is initiated with (1a) followed by (1b) and (1a) etc. This makes the "p" sequence never to be exposed to the initial condition of $q_{0}$ as in $\mathrm{q}_{1}\left(\mathrm{p}_{0}, \mathrm{q}_{0}\right), \mathrm{p}_{1}\left(\mathrm{p}_{0}, \mathrm{q}_{1}\right), \mathrm{q}_{2}\left(\mathrm{p}_{1}, \mathrm{q}_{1}\right), \mathrm{p}_{2}\left(\mathrm{p}_{1}, \mathrm{q}_{2}\right) \ldots$ The sequence is iterated 200 times and then, depending on the requirements, either the next 200 iterations or the immediately following value collected. As (1a) is used in the first iteration, the two sequences are asymmetric with the Q-sequence having an advantage of the first move.

The basin of attraction chart is obtained by setting an equally spaced 1,000x1,000 grid of initial conditions covering the square $\left(0,0, \mathrm{p}_{\max }, \mathrm{q}_{\max }\right)=(0,0,1.2,1.2)$. Starting from each initial point, a trajectory is calculated and then evaluated at $\mathrm{k}=2.05$. 


\section{Two Parameter Chaotic Entropy}

Let us describe an interaction between two fields, both described by the Boltzmann-Gibbs entropies which are understood as probabilities and interact with each other through the standard conditional relationships.

$$
\begin{aligned}
& q_{n+1}=P(q \mid p)=\frac{P(q \cap p)}{P(p)}=\frac{-k q_{n} p_{n} \ln \left(q_{n} p_{n}\right)}{p_{n}}=-k q_{n} \ln \left(q_{n} p_{n}\right) \\
& p_{n+1}=P(p \mid q)=\frac{P(q \cap p)}{P(q)}=\frac{-k q_{n} p_{n} \ln \left(q_{n} p_{n}\right)}{q_{n}}=-k p_{n} \ln \left(q_{n} p_{n}\right)
\end{aligned}
$$

(Note: Here, $k$ stands for a parameter that varies between 0 and the Euler's number and does not relate to the Boltzmann constant.) Formulae (1a) and (1b) constitute a two-dimensional set of feedback sequences, which is highly dependent on initial conditions $\mathrm{p}_{0}$ and $\mathrm{q}_{0}$. An example of trajectories obtained through (1a)(1b) for a selection of randomly selected initial conditions is shown in Figure 1. At $\mathrm{k}$ between 1.5 and 2, the trajectories diverge to one of the extremes ( 0 or 1 ). This is followed by a period of being constant and then attaining chaos as the later phase.

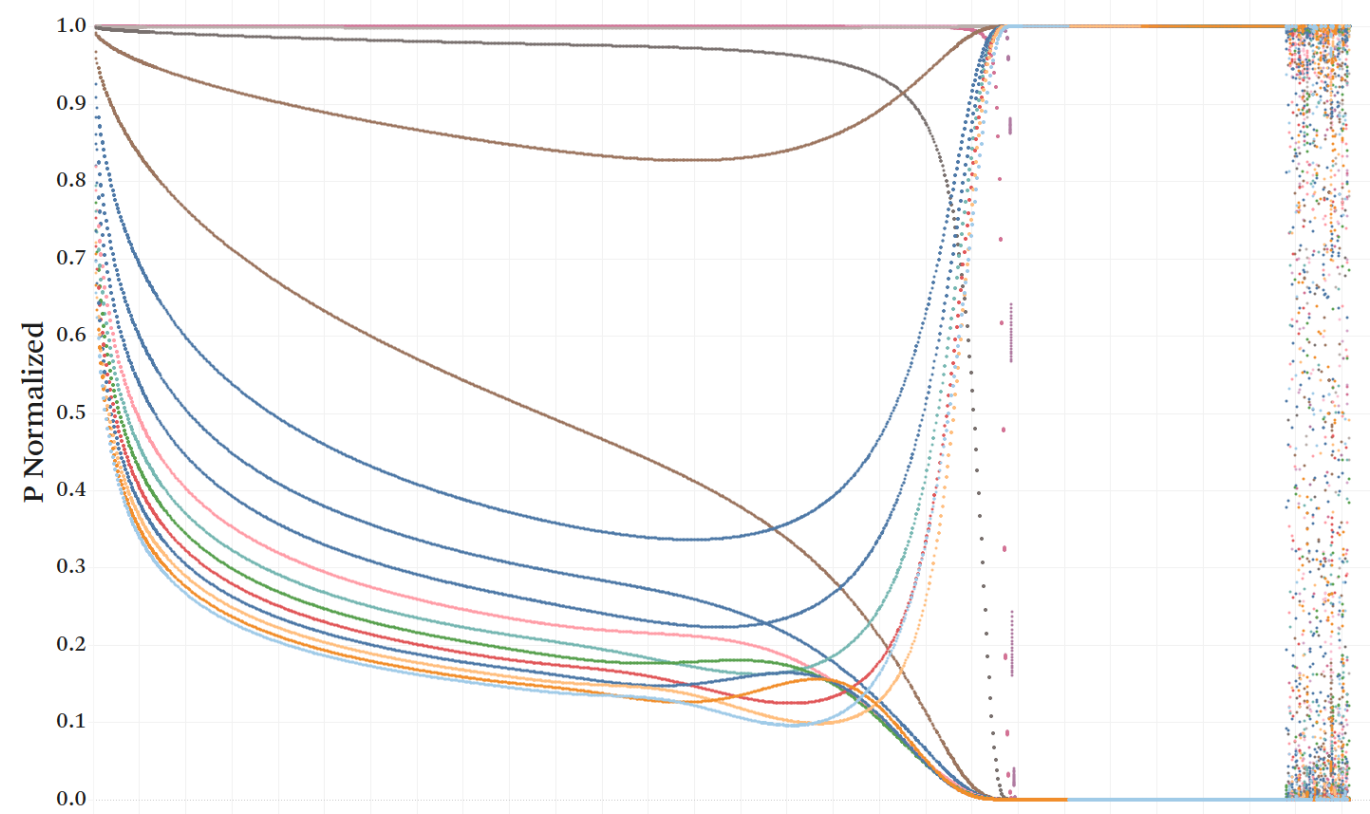

$\begin{array}{lllllllllllllllllllllllllllll}0.1 & 0.2 & 0.3 & 0.4 & 0.5 & 0.6 & 0.7 & 0.8 & 0.9 & 1.0 & 1.1 & 1.2 & 1.3 & 1.4 & 1.5 & 1.6 & 1.7 & 1.8 & 1.9 & 2.0 & 2.1 & 2.2 & 2.3 & 2.4 & 2.5 & 2.6 & 2.7 & 2.8\end{array}$

Figure 1. An example of p-normalized trajectories

\section{The uncertainty principle}

The central prerequisite of the method employed here is the uncertainty principle. The presence of uncertainly results in trajectories forming distinct probability levels and influences the calculation of composite particle masses. In classical quantum mechanics, uncertainty is intrinsically associated with the properties of wave functions. The method presented here does not employ wave functions therefore uncertainty must be arrived at through a different argument.

As is evident from Figure 1, at $\mathrm{k}$ in the 2.0 to 2.4 range all trajectories arrive at normalized values of either 0 or 1 . This is interpreted as corresponding to spin in either up or down alignment. An assignment of specific values to an attractor splits the basin of attraction into distinctive bands with initial conditions resulting in trajectories leading towards one of these two states. Figure 2 illustrates this phenomenon showing the distinctive banding of the basin of attraction. Here, any initial condition within a green band attains 1 for values of $\mathrm{k}$ in $(2.0,2,4)$ and the initial conditions within the yellow bands attain 0 for such values of $\mathrm{k}$. 


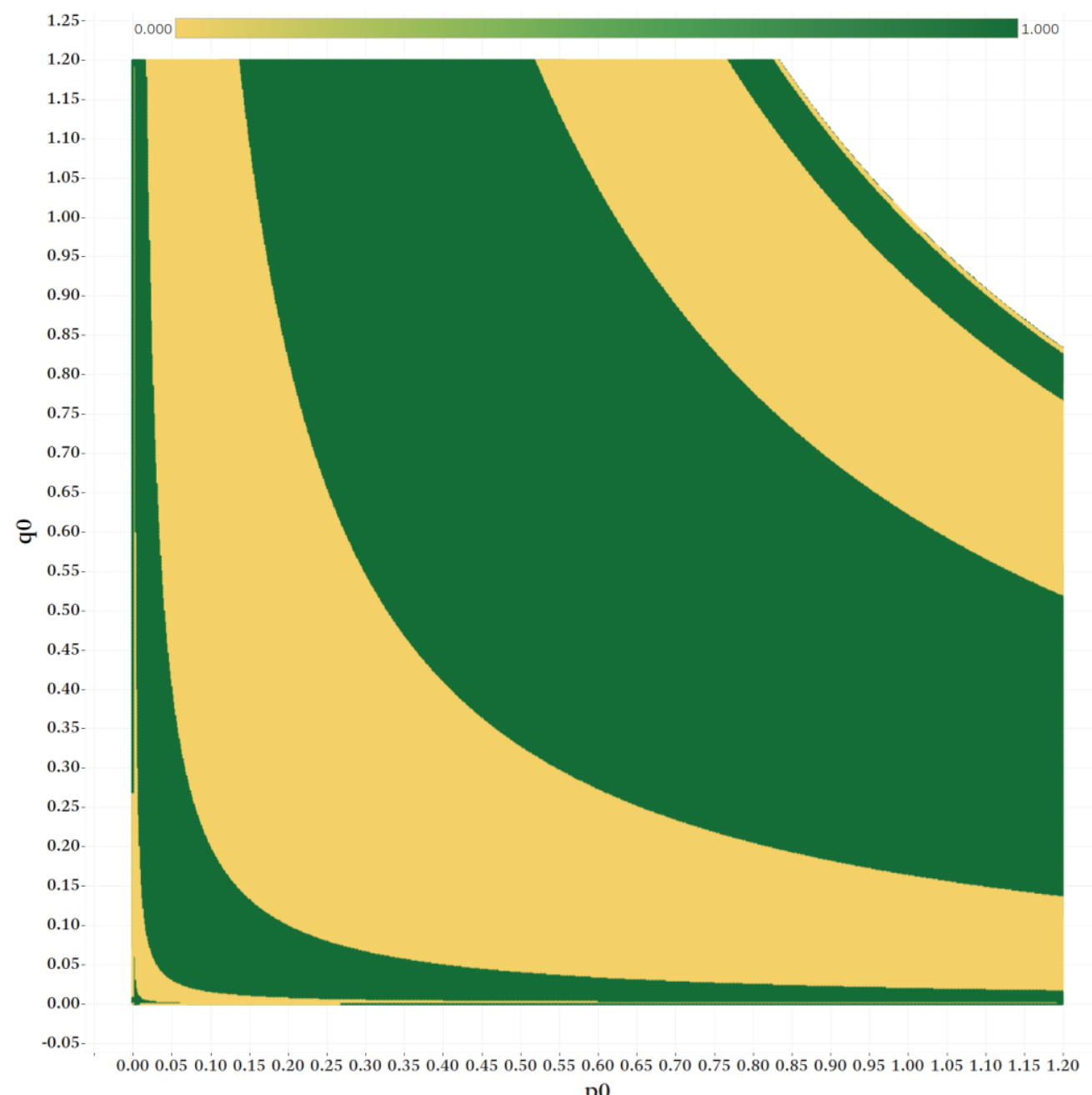

p0

Figure 2. Close up of basin of attraction at $\mathrm{k}=2.1$ and $\mathrm{p}_{0}$, $\mathrm{q}_{0}$ under 1.20 showing attractor bands

The uncertainty principle is introduced by observing that from the spins' point of view it is impossible to establish what point within a band was used as the initial condition to arrive at this spin value. Thus, for example, all points in the central green band would produce the "up" value as long as the initial point is within that band.

The $\mathrm{p}_{0}=\mathrm{q}_{0}$ line cuts the bands in their widest region allowing for the maximal width of each band to be measured. This widest width is used as the measure of the uncertainty associated with each band, providing a one-dimensional uncertainty defining parameter. This parameter is applied to the values of $\mathrm{p}, \mathrm{q}$ and $\mathrm{k}$ variables. Thus, when performing a computation, the sequence is calculated at the highest precision available. However, the values used to obtain the normalised $\mathrm{p}, \mathrm{q}$ and $\mathrm{k}$ are scaled through the uncertainty parameter as in (2abcde). In the case of $q$ and $p$ sequences, the integer portion of the scaling is taken so to implement rounding. $\mathrm{K}$ is set as multiples of the width of each band splitting the attractor into a collection of distinctive points. (q-sequence is shown, p-sequence is similar)

$$
\begin{gathered}
q_{\text {integer }}=\operatorname{Integer}\left(\frac{q}{(\text { width of band })}\right) \\
q_{\text {scaled }}=q_{\text {integer }} *(\text { width of band }) \\
k_{i}=(\text { width of band }) * i
\end{gathered}
$$


Thus, the computed number is divided by the width of the band, converted to an integer, and therefore truncated, and then multiplied back by the width of the band to arrive at the scaled value. The scaled value is used to find $\mathrm{q}$ and $\mathrm{p}$ normalized. The attractor charts are plotted for the normalized values of $\mathrm{p}$-normalized and q-normalized against granulated $\mathrm{k}$ as in $(2 \mathrm{c}),(2 \mathrm{~d})$ and $(2 \mathrm{e})$.

$$
\begin{aligned}
& q \text { normalised } \stackrel{\text { def }}{=} \frac{q_{\text {scaled }}}{q_{\text {scaled }}+p_{\text {scaled }}} \\
& p \text { normalised } \stackrel{\text { def }}{=} \frac{p_{\text {scaled }}}{p_{\text {scaled }}+q_{\text {scaled }}}
\end{aligned}
$$

Table 1 lists each band characteristics as measured by setting the trajectories' initial conditions to $\mathrm{p}_{0}=\mathrm{q}_{0}$.

Table 1. Widths of attractor basin bands

\begin{tabular}{lllll} 
Band Id & Lower Edge $\mathrm{p}_{0}$ & Upper Edge $\mathrm{p}_{0}$ & Spin & Width of Band \\
\hline 1 & 0.00000001000 & 0.00000001086 & 0 & 0.00000000086 \\
2 & 0.00000001129 & 0.00000009492 & 1 & 0.00000008363 \\
3 & 0.00000009535 & 0.00000078541 & 0 & 0.00000069007 \\
4 & 0.00000078584 & 0.00000603617 & 1 & 0.00000525033 \\
5 & 0.00000603660 & 0.00004288499 & 0 & 0.00003684839 \\
6 & 0.00004288542 & 0.00027871100 & 1 & 0.00023582558 \\
7 & 0.00027872700 & 0.00163415600 & 0 & 0.00135542900 \\
8 & 0.00163423800 & 0.00848224000 & 1 & 0.00684800200 \\
9 & 0.00848412000 & 0.03797004000 & 0 & 0.02948592000 \\
10 & 0.03797192000 & 0.14073648000 & 1 & 0.10276456000 \\
11 & 0.14073836000 & 0.40388424500 & 0 & 0.26314588500 \\
12 & 0.40389073800 & 0.78812363500 & 1 & 0.38423289700 \\
13 & 0.78813012700 & 0.95861575200 & 0 & 0.17048562500 \\
14 & 0.95861620500 & 0.99524989500 & 1 & 0.03663369000 \\
15 & 0.99525034700 & 0.99965653100 & 0 & 0.00440618400 \\
16 & 0.99965657900 & 0.99998285400 & 1 & 0.00032627500 \\
17 & 0.99998290100 & 0.99999936400 & 0 & 0.00001646300 \\
18 & 0.99999936400 & 0.99999998100 & 1 & 0.00000061700 \\
19 & 0.99999998200 & 0.99999999900 & 0 & 0.00000001700
\end{tabular}

For the attractor to move along increasing $\mathrm{k}$, the uncertainty introduced gaps need to be traversed, so to continue the movement. This is perceived as resistance. Therefore, the phenomenon interpreted as "mass" is a manifestation of discontinuity in probability. Alternatively, one can interpret "mass" as a discontinuity in entropy distribution. Figure 3 illustrates trajectories for bands 4, 5 and 6 in the region of small $\mathrm{k}$. 


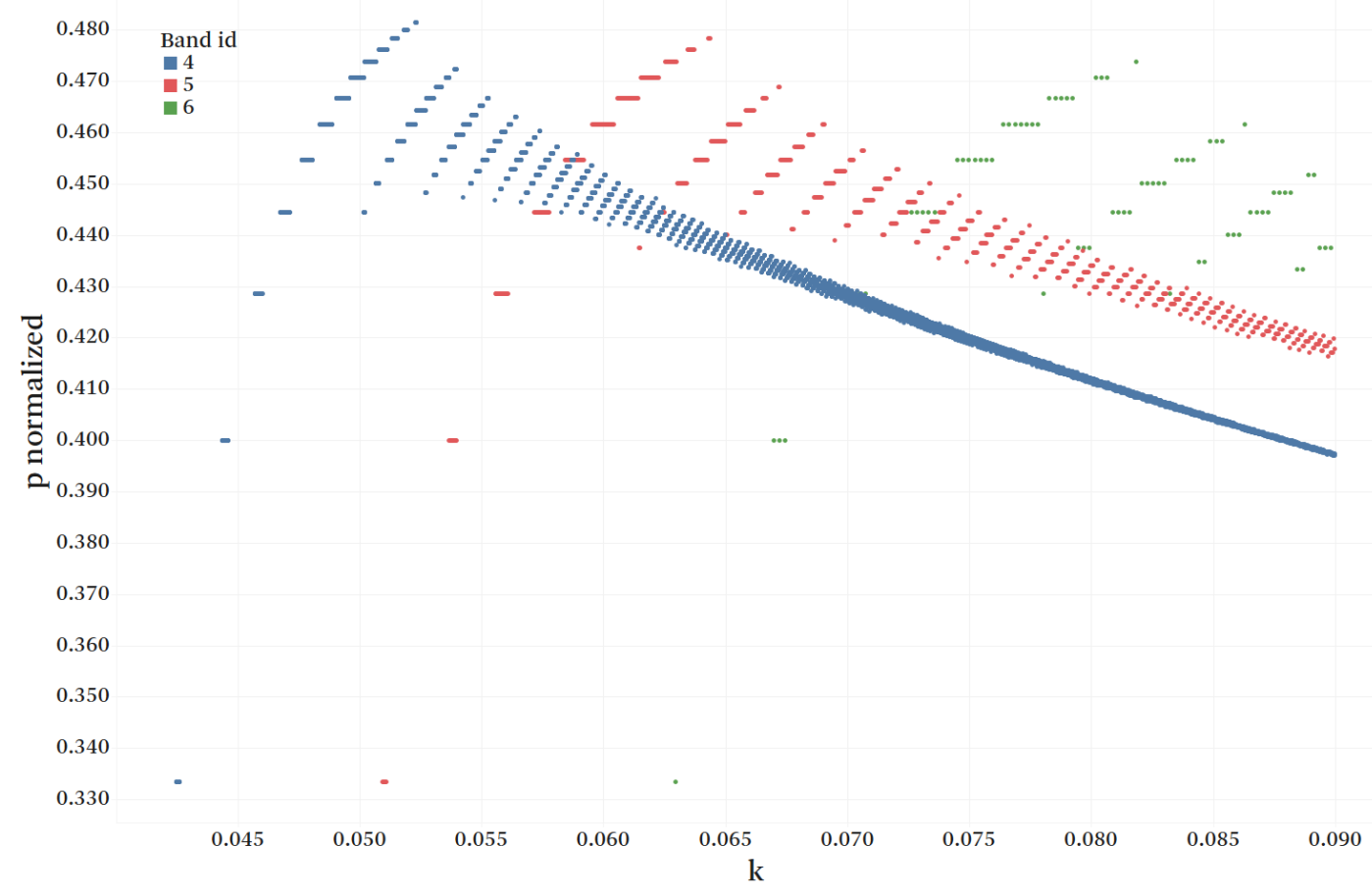

Figure 3. Trajectories for bands 4, 5 and 6 . The central point of each band was used as the initial condition for each trajectory.

\section{The Probability Field and the Quark Masses}

The following is an application of the modelling method as in (1a) (1b) which allows for retrieving of the quark masses. The initial conditions are set to $\mathrm{q}_{0}=\mathrm{p}_{0}$ for each trajectory with values set in the middle of each band. In Figure 4 the trajectory for the $4^{\text {th }}$ band is plotted and the locations of the lines relevant to the quark positions are indicated.

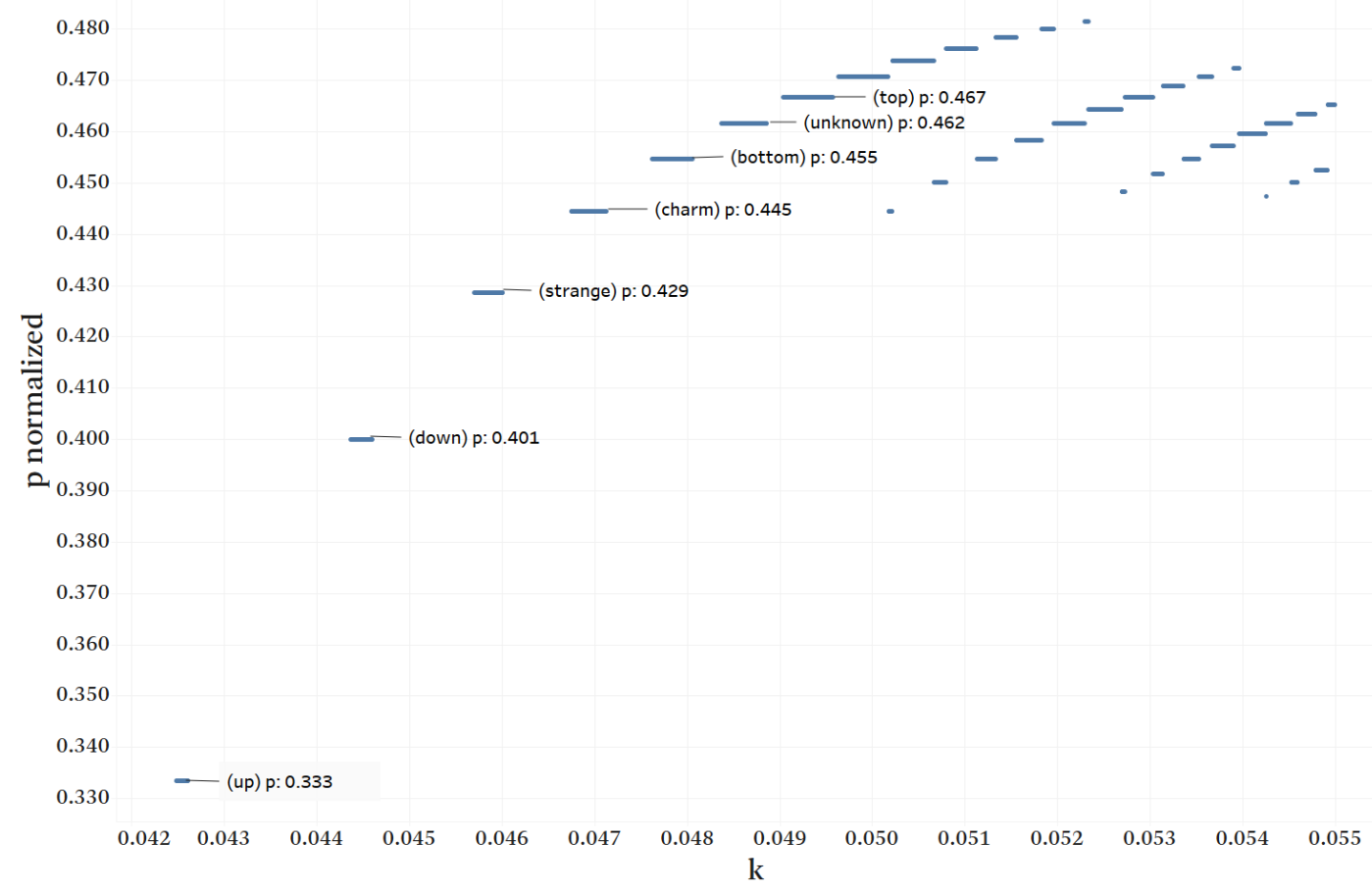

Figure 4. The trajectory for band 4 with quark levels 
It is assumed that gaining in mass is achieved by transiting from each line to the line above. The change in probability is denoted as $\Delta$ line. These changes in probability are associated with the mass through (3a) (3b), which show that the gain in mass is exponentially proportional to the inverse of the square root of the probability step.

$$
\begin{gathered}
M=M_{0} e^{\frac{1}{\sqrt{\Delta l i n e}}} \\
\text { where } M_{0}=0.15014817 \frac{\mathrm{MeV}}{c^{2}}
\end{gathered}
$$

Formula (3a) has not been derived but rather it is proposed. The authors arrived at this relationship by looking at the chart characteristics and the way the data aligns itself. The scaling constant $\mathrm{M}_{0}$ is found by setting $\Delta$ line $=2 / 3$ and assuming that such transition corresponds to the mass of an electron, which is known to eight decimal places.

Table 2 compares quark masses found through the model to those that are experimentally measured. The experimental data is quoted as published by the Particle Data Group. Except for the down quark and the top, the values proposed by the model undershoot those found experimentally. The difference is indicated in the deviation column.

If the model is correct, one would expect to find the top at much lower energy and to assign the level found experimentally to an excited state. Otherwise, if the experimentally found resonance is indeed the top then the model would expect an additional quark of the mass of about 25,000 MeV/c ${ }^{2}$. While taking into account the negative bias of the data-model relationship this mass has been indicated as bracketed at ID 6 . There is also room for higher energy states as indicated in the table.

Table 2. The Model and the Experimental results (mass is in $\mathrm{MeV} / \mathrm{c}^{2}$ )

\begin{tabular}{cccccccc} 
ID & Start & End (quark level) & Step & Model & Experimental & Deviation & Quark \\
\hline 1 & 0.0000000 & 0.3333333 & 0.3333333 & 0.85 & 2.16 & $-60.7 \%$ & Up \\
2 & 0.3333333 & 0.4000000 & 0.0666667 & 7.22 & 4.67 & $54.6 \%$ & Down \\
3 & 0.4000000 & 0.4285714 & 0.0285714 & 55.70 & 93 & $-40.1 \%$ & Strange \\
4 & 0.4285714 & 0.4444444 & 0.0158730 & 420 & 1,270 & $-66.9 \%$ & Charm \\
5 & 0.4444444 & 0.4545455 & 0.0101010 & 3,146 & 4,180 & $-24.7 \%$ & Bottom \\
6 & 0.4545455 & 0.4615385 & 0.0069930 & 23,438 & $(28,791)$ & & Unknown \\
7 & 0.4615385 & 0.4666667 & 0.0051282 & 174,226 & 172,760 & $0.8 \%$ & Top \\
8 & 0.4666667 & 0.4705882 & 0.0039216 & $1.293 \mathrm{E}+06$ & & & \\
9 & 0.4705882 & 0.4736842 & 0.0030960 & $9.588 \mathrm{E}+06$ & & &
\end{tabular}

Figure 5 compares experimentally found quark masses with the values proposed by the model. The chart employs a logarithmic scale which produces a straight line for the model. 
$1,000,000$

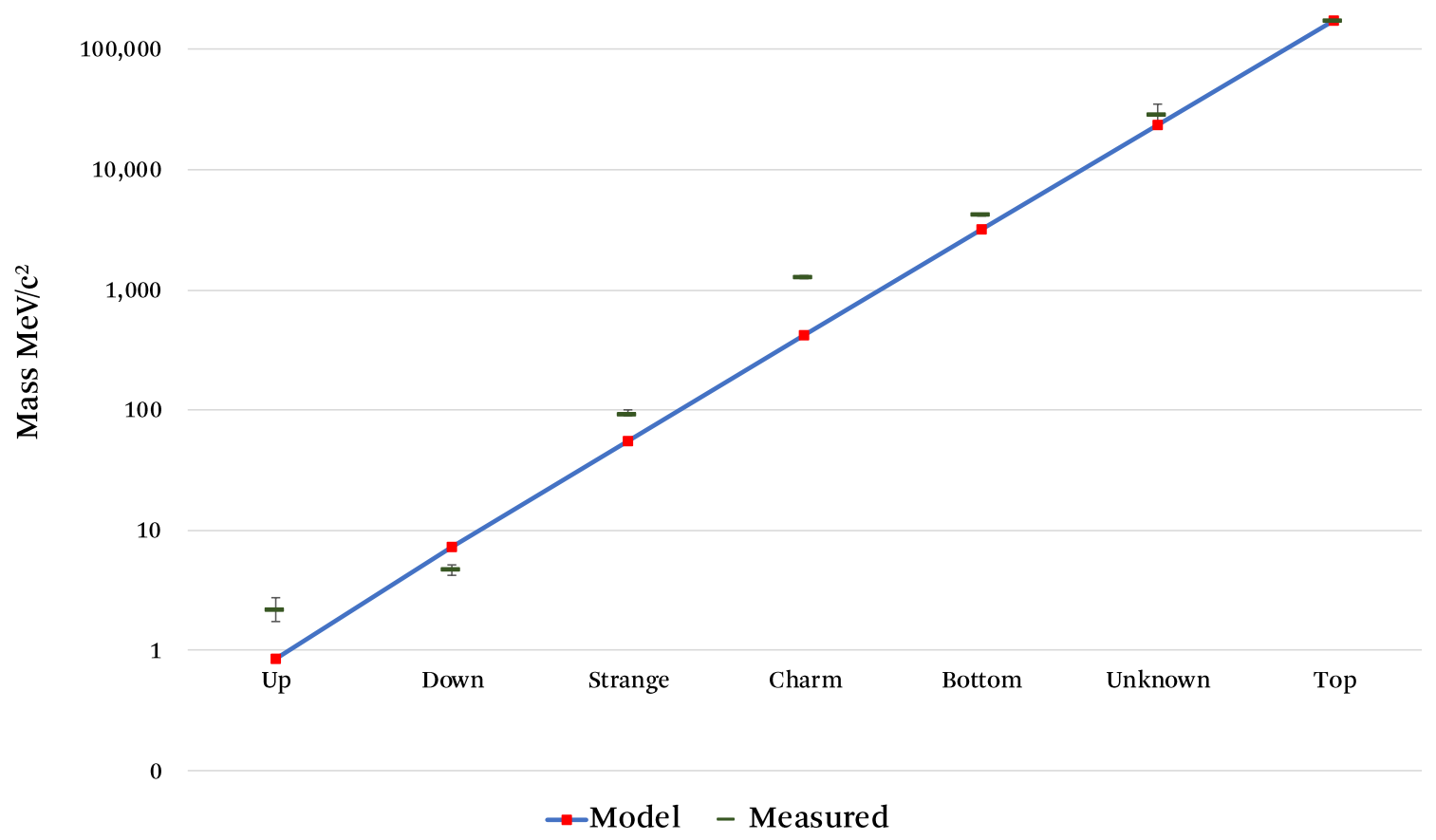

Figure 5. Reported and Modelled Quark Masses

There is an obvious discrepancy between the model and the experimental data. A somewhat unsatisfactory remedy would be to look for enhanced measurement precision. On the other hand, one could look for a process similar but opposite to the one observed in nucleus formation, where binding energy results in the mass of constituents being larger than the mass of the resultant nucleus. In the case of quarks, the presence of gluonic force could increase the measured mass of constituents when compared to their theoretical values. This would be consistent with the peculiarities of gluonic forces when compared to other known interactions.

\section{Baryon masses and weak interactions}

Bands 4, 5 and 6 allow for the reproduction of the baryon masses. Band 7 does not contain the up quark due to the width of the band. Table 3 summarises values obtained here and compares these to the experimental data published by the Particle Data Group. A percentage deviation between the model and the experimental results is presented in the last column.

Table 3. Summary of the results

\begin{tabular}{lrrrr} 
Particle & Constituents & Model $\left(\mathrm{MeV} / \mathrm{c}^{2}\right)$ & Experimental $\left(\mathrm{MeV} / \mathrm{c}^{2}\right)$ & Difference $(\%)$ \\
\hline proton & uud & 938.394 & 938.272 & $0.013 \%$ \\
neutron & udd & 939.897 & 939.565 & $0.035 \%$ \\
$\Sigma$ & uus & $1,189.431$ & $1,189.370$ & $0.005 \%$ \\
$\Sigma$ & uds & $1,195.802$ & $1,192.642$ & $0.265 \%$ \\
$\Sigma$ & dds & $1,202.174$ & $1,197.449$ & $0.395 \%$ \\
W Boson & W & $79,920.362$ & $80,379.000$ & $-0.571 \%$
\end{tabular}

The following is the presentation of the method as applied to each calculation.

\section{Gluons and the probability metric}

Quarks interact through the exchange of gluons. This is represented here through a metric (4a) which assumes that the interaction requires a gluons' movement along k-direction and p-direction, both treated as 
changes in probability, with the absolute value of the product measuring the amount of the movement. The effective probability is the mean of the movements $(4 b)$.

$$
\begin{gathered}
\Delta_{i j}=\left|\left(k_{j}-k_{i}\right)\left(p_{j}-p_{i}\right)\right| \\
\Delta \text { line }=\frac{1}{2} \sqrt{\frac{\sum \Delta_{\mathrm{ij}}}{\mathrm{N}}}
\end{gathered}
$$

In (4b) the summation is over all possible movements and $\mathrm{N}$ stands for the number of combinations. In the case of strange baryons, which require four movements, (4a) contains four terms (two k and two p). In that instance, a square root is taken of the product to recover the second power of probability in (4a).

\section{The composite band error}

The calculations are subject to still another uncertainty related principle. A baryon consists of three quarks. Each quark is distinct as it is associated with a separate band. The quarks can interact with each other in six ways $(12)(13)(23)(21)(31)(32)$. Each interaction involves two moves, one along the k-direction and another along the p-direction as in (4a). Therefore, when calculating the $\Delta$ line, there are 12 possible sources of error.

Each movement is subject to an error associated with the width of the band. This error is calculated as the geometric mean of the three widths. Therefore, the maximum error allowed by the movements is 12 times the geometric mean of the widths. This puts maximum value on the probability of composite particles. Table 4 compares the sum of the quark probabilities (listed in Table 2 in the column labelled as "End") with the maximum error allowed.

\begin{tabular}{|c|c|c|c|c|}
\hline Particle & Quarks & Sum of Quark Levels (End) & $1+$ Max Error & Difference \\
\hline proton & uud & 1.067 & 1.072 & -0.005 \\
\hline neutron & udd & 1.133 & 1.072 & 0.062 \\
\hline$\Sigma$ & uus & 1.095 & 1.072 & 0.024 \\
\hline$\Sigma$ & uds & 1.162 & 1.072 & 0.090 \\
\hline$\Sigma$ & dds & 1.229 & 1.072 & 0.157 \\
\hline
\end{tabular}

Table 4. Composite band error and the particle constituents' probabilities

Table 4 shows that only the proton's total probability imposed through the constituents is less than the maximum error allowed. All other baryons exceed this error which influences the stability of those particles. This has two consequences. Firstly, the life span of the particle is influenced, with the proton being the only truly stable. Secondly, the excess of probability must be redirected somewhere for the particle to exist. 


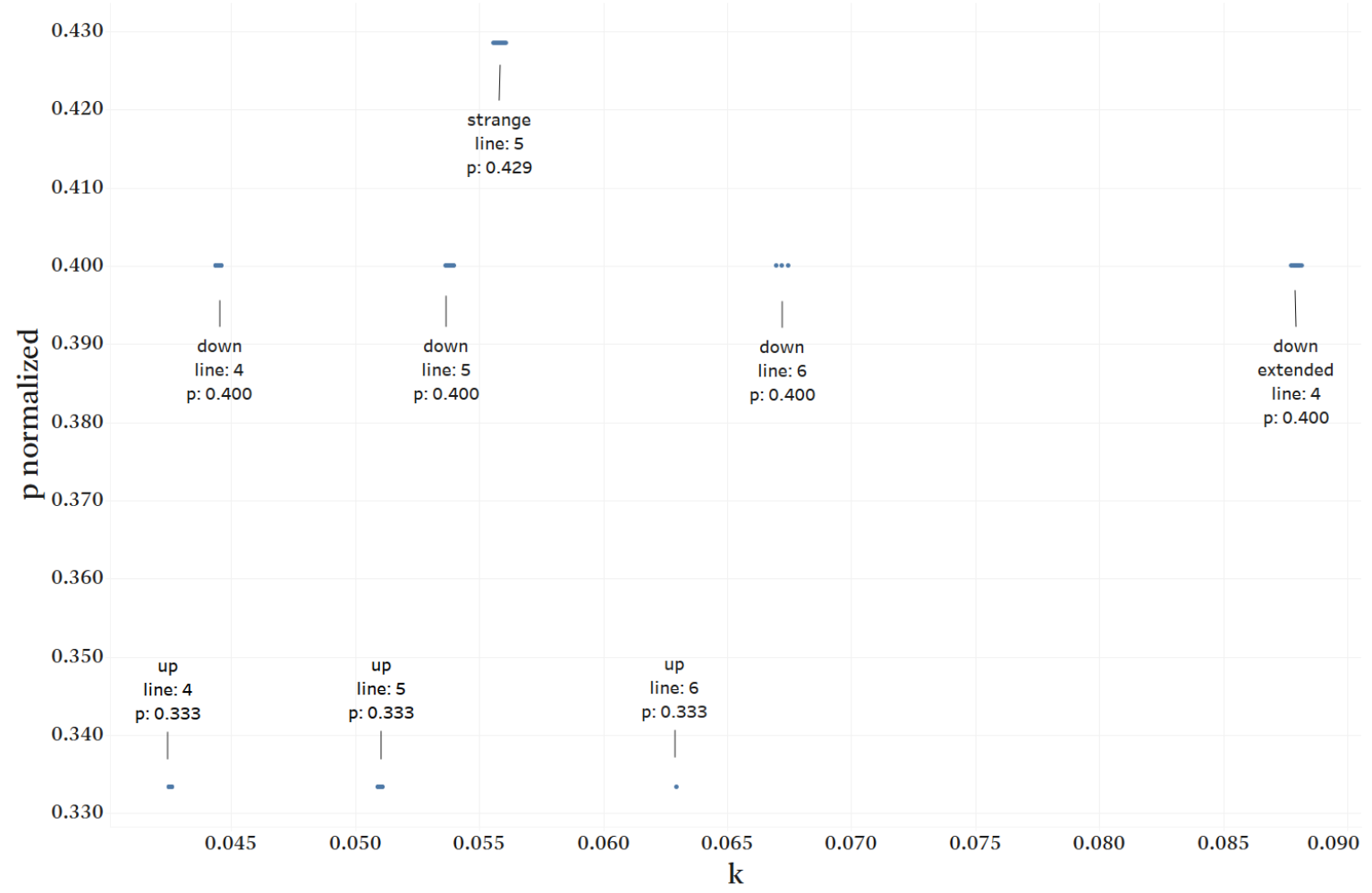

Figure 6. Quark lines/trajectories 4, 5 and 6 for up, down and strange quarks as used in calculations

Granulation in the $\mathrm{k}$ direction is introduced through (2c). The width of $\mathrm{k}$ spacing is dependant on the band considered, with the $4^{\text {th }}$ band producing the least spaced points and the $6^{\text {th }}$ band the most spread out. The number of points is also influenced by granulation. Table 5 lists the number of points on each level as in Figure 6. Only those involved in the subsequent calculations are shown. In the case of the neutron, the down quark on the $4^{\text {th }}$ trajectory includes the 21 extended points as in Figure 6.

Table 5. Point counts for levels as in Figure 6

\begin{tabular}{|c|c|c|c|c|c|c|c|c|c|c|c|c|}
\hline & \multicolumn{3}{|c|}{ W } & \multicolumn{3}{|c|}{ neutron } & \multicolumn{3}{|c|}{ proton } & \multicolumn{3}{|c|}{ sigma } \\
\hline & 4 & 5 & 6 & 4 & 5 & 6 & 4 & 5 & 6 & 4 & 5 & 6 \\
\hline s - 0.42857 & & & & & & & & & & & 15 & \\
\hline $\mathrm{d}-0.40000$ & 44 & 10 & 3 & $44+21$ & & 3 & & 10 & & & 10 & \\
\hline $\mathrm{u}-0.33333$ & 25 & 6 & 1 & & 6 & & 25 & & 1 & 25 & 6 & 1 \\
\hline
\end{tabular}

\section{Proton $-u_{4} d_{5} u_{6}$}

Proton is obtained by placing the first up quark on the $4^{\text {th }}$ band, the down quark on the $5^{\text {th }}$ band and the second up quark on the $6^{\text {th }}$ band. The number of gluon combinations $\mathrm{N}$ is calculated as a sum of pairwise products, giving $10 * 1+10 * 25+25 * 1=285$. The line width is calculated with $(4 \mathrm{a})$. These are entered into (4b) and (3a). To obtain the result listed in Table 4 the proton quark-constituent masses of two up quarks and one down quark are subtracted from (3a).

$$
\text { proton mass }=M-2 m_{u}-m_{d}
$$

In all calculations, the model calculated quark masses as listed in Table 2 are used.

\section{Neutron $-d_{4} u_{5} d_{6}$ including the $4^{\text {th }}$ band which is expanded}

Neutron is obtained by placing the first down quark on the $4^{\text {th }}$ band, the up quark on the $5^{\text {th }}$ band and the second down quark on the $6^{\text {th }}$ band. The $4^{\text {th }}$ band is expanded to include points on the closest returning curve as 
in Figure 3 and Figure 6 ( $\mathrm{k}$ in the region of 0.088). The expansion is to partially accommodate the excess of probability. The remaining excess of probability modifies the method of combinations counting which are increased by the maximum amount of the available error. The combinations $\mathrm{N}$ are counted as: $\mathrm{N}=\left(65^{*} 6+65^{*} 3\right.$ $+6 * 3)^{*} 1.071732166=646.254$

The redirection of the excess in probability consists of two processes. Firstly, the neutron increases the gluonic activity through the utilization of the measurement error, but only to its maximum value. In this setting, gluons are more active than the ordinary combination count would have indicated. The remaining excess of probability expands the $\mathrm{k}$ range so to include the points on the returning branch of the trajectory. The closest points, which are on the $4^{\text {th }}$ band are therefore included.

To obtain the line width the summing is calculated over points that include the expanded $4^{\text {th }}$ band. These together with the expanded combinations count is entered into (4b) and (3a).

To obtain the result listed in Table 4 the neutron quark-constituent masses of two down quarks and one up quark are added to (3a). As in the case of the proton, the model calculated quark masses are entered in (6).

$$
\text { neutron mass }=M+m_{u}+2 m_{d}
$$

\section{Sigma - uus, uds, dds set within the u-d-s context}

Sigma requires a modified calculation procedure due to the strange quark being separated from the lower quark levels. As in the case of the proton and the neutron, the mass of the composite particle is obtained through (3a) and then adjusted, similarly to the neutron, by adding the masses of quark-constituents.

The line width is calculated by assuming that the points belonging to the $\mathrm{d}_{5}$ quark location act as intermediaries in communication between all three up-quark lines $\left(\mathrm{u}_{4}, \mathrm{u}_{5}\right.$ and $\left.\mathrm{u}_{6}\right)$ and the central strange quark $\mathrm{s}_{5}$. The sum is run over the full collection of the up and strange quark points. The individual line movements are calculated as in (7) with the sum running over the intermediate $\mathrm{d}_{5}$ quark points.

$$
\Delta_{i j}=\sqrt{\sum_{n}\left|\left(k_{j}-k_{n}\right)\left(k_{n}-k_{i}\right)\left(p_{j}-p_{n}\right)\left(p_{n}-p_{i}\right)\right|}
$$

The pairwise combination counting is done over the entire set of points giving 1,425 possible moves as shown in Table 6. This count is multiplied by two factors. The first factor is the available error, which is the same as the one used in the case of the neutron. The second factor is derived from the error in $\mathrm{k}$.

\begin{tabular}{|c|c|c|c|c|c|c|c|c|c|c|}
\hline Level & Band & Points & \multicolumn{8}{|c|}{ Combinations $($ sum $=1,425)$} \\
\hline 0.3333 & 4 & 25 & & & & & & & & \\
\hline 0.4000 & 4 & 0 & 0 & & & & & & & \\
\hline 0.4286 & 4 & 0 & 0 & 0 & & & & & & \\
\hline 0.3333 & 5 & 6 & 150 & 0 & 0 & & & & & \\
\hline 0.4000 & 5 & 10 & 250 & 0 & 0 & 60 & & & & \\
\hline 0.4286 & 5 & 22 & 550 & 0 & 0 & 132 & 220 & & & \\
\hline 0.3333 & 6 & 1 & 25 & 0 & 0 & 6 & 10 & 22 & & \\
\hline 0.4000 & 6 & 0 & 0 & 0 & 0 & 0 & 0 & 0 & 0 & \\
\hline 0.4286 & 6 & 0 & 0 & 0 & 0 & 0 & 0 & 0 & 0 & 0 \\
\hline
\end{tabular}

Table 6. Calculation of combinations for the Sigma structure

In the case of the Sigma, the widths introduced through the $\mathrm{k}$ spread are used as the second factor to accommodate the probability excess. Table 7 lists the k-direction error. The largest values of k-width for each band are added together and multiplied by 3 to accommodate the presence of 3 quarks in the system. Therefore the effective combinations are $1,425 * 1.071732 * 1.003640=1532.777$ 
Table 7. Width of k related uncertainty for the Sigma structure

\begin{tabular}{ccccc} 
Quark Line & Min of k & Max of k & k-width & Largest \\
\hline Band 4 & & & \\
0.3333 & 0.042486 & 0.042612 & 0.000126 & \\
0.4000 & 0.044371 & 0.044596 & 0.000226 & 0.000226 \\
\hline Band 5 & & & \\
0.4000 & 0.053651 & 0.053983 & 0.000332 & 0.000516 \\
\hline 0.4286 & 0.055567 & 0.056083 & 0.000516 & \\
\hline Band 6 & & & 0.000472 \\
\hline 0.4333 & 0.062965 & 0.062965 & 0.000000 & 0.001213 \\
\hline & 0.066974 & 0.067446 & 0.000472 & 1.003640 \\
\hline
\end{tabular}

To obtain the mass of Sigma, M is calculated using (3a), then multiplied by 2 and the masses of constituent quarks are added. In (8) 1,2 and 3 indexes stand for the strange and the relevant combination of up and down quarks.

$$
\Sigma_{\text {mass } 123}=2 M+m_{q 1}+m_{q 2}+m_{q 3}
$$

The factor of 2 in (8) is understood to account for the strange quark being two levels above the up quark location.

\section{The W Boson u4-d4, u5-d5 and u6-d6 transitions}

The mass of the $\mathrm{W}$ boson is obtained by limiting the interactions to those which are within each one of the three bands. Thus, only $\mathrm{u}_{4}-\mathrm{d}_{4}, \mathrm{u}_{5}-\mathrm{d}_{5}$ and $\mathrm{u}_{6}-\mathrm{d}_{6}$ transitions are considered. The line width is calculated using (4a) for each transition band independently, and then the results added together to represent the entire structure. Similarly, the number of combinations is arrived at by adding combinations for each of the bands separately, that is $(44 * 25+10 * 6+3 * 1)=1,163$. Formula $(4 \mathrm{~b})$ is employed to calculate the effective width and (3a) to arrive at the mass listed in Table 3 . No other factors are applied in the calculation.

\section{Discussion and Conclusion}

The paper presents a model of intra-entropic interactions utilizing the Boltzmann-Gibbs entropy formula. The methods of chaos theory are employed requiring a direct association of entropy with the probability to obtain feedback formulae that may be iterated. Therefore, in this understanding, probability and entropy are equivalent. The existence of a probability field is postulated, with the field manifesting its presence by influencing the structure of the physical world.

The assumption of the presence of a probability field is used to recover quark masses. In this case, it is anticipated that the number of quarks occurring in nature is larger than that proposed by the Standard Model. As the proposed quark masses are well defined, experimental verification of the model is possible.

The composite particle masses are arrived at through an introduction of a metric that assumes that interaction consists of at least two probability moves. One along the k-direction and the other along the p-direction. Thus, states do not interact directly but need to find paths to exchange gluons. Combinatoric counting is used to find the effective line distance. The necessity to find a path of interaction is most evident in the case of Sigma where all interactions are channelled through the $\mathrm{d}_{5}$ quark location. It is assumed that $\mathrm{d}_{5}$ quark is not physically present in this arrangement, but its position and individual points are utilized to facilitate the gluon transmission.

Each quark is associated with a probability. In the case of a composite particle, these when added together exceed unity. There is also an error tolerance due to the widths of the bands which provides for the maximum allowed excess of the composite probability. Thus, in the case of the proton, the composite probability is within the allowed error region, making this particle permanent.

Other baryons exceed the allowed error and therefore their lifetimes are reduced. In any of those cases, the particle allocates the excess in probability into some additional arrangement. The most direct way is to increase 
the activity of gluons, which can be increased only up to the available band-related error. The second method, which is employed by the neutron, is to enlarge the number of points on the $4^{\text {th }}$ trajectory. In the case of the Sigma, the particle is confined to the low-k quark lines, and it seeks to consume the remaining excess in probability by utilizing the k-position error.

\section{Data Availability}

The data that support the findings of this study are available from the corresponding author upon reasonable request.

\section{References}

1. Caticha, A. Entropic Dynamics. Entropy 2015, 17(9), 6110-6128; https://doi.org/10.3390/e17096110

2. Shannon, C. E. Probability of error for optimal codes in a Gaussian channel, Bell Syst. Tech. J., vol. 38, pp. 611-656, May 1959.

3. Verdu, S. Fifty years of Shannon theory, in IEEE Transactions on Information Theory, vol. 44, no. 6, pp. 2057-2078, Oct. 1998, https://doi.org/10.1109/18.720531

4. Martyushev, L.M.; Shaiapin, E.V. Entropic Measure of Time, and Gas Expansion in Vacuum. Entropy 2016, 18, 233. https://doi.org/10.3390/e18060233

5. Caticha, Ariel, Entropic Time, AIP Conference Proceedings 1305, 200-207 (2011); https://doi.org/10.1063/1.3573617

6. Martyushev, L.M. On Interrelation of Time and Entropy. Entropy 2017, 19(7), 345; https://doi.org/10.3390/e19070345

7. Vilenchik, L. and Vilenchik, M. (2019) The Emergence and Evolution of the Universe. Journal of High Energy Physics, Gravitation and Cosmology, 5, 884-898. https://doi.org/10.4236/jhepgc.2019.53044

8. Wissner-Gross, A. D., and C. E. Freer. Causal Entropic Forces. Physical Review Letters 110.16 (2013). http://hdl.handle.net/1721.1/79750

9. Feigenbaum, M. (1978), Quantitative universality for a class of non-linear transformations, J. Statist. Phys. 19 , 25-52.

10. Luque, B., Lacasa, L., Ballesteros, F. J., \& Robledo, A. (2011). Feigenbaum graphs: a complex network perspective of chaos. PloS one, 6(9), e22411. https://doi.org/10.1371/journal.pone.0022411

\section{To cite this article}

\section{APA}

Szweizer, M., \& Schlagbaum, R. (2021, February 27). Probability Field in Chaotic Intra-Entropic Interactions. https://doi.org/10.31219/osf.io/y5v6d

\section{MLA}

Szweizer, Moshe, and Rivka Schlagbaum. "Probability Field in Chaotic Intra-entropic Interactions." OSF Preprints, 27 Feb. 2021. Web.

\section{Chicago}

Szweizer, Moshe, and Rivka Schlagbaum. 2021. "Probability Field in Chaotic Intra-entropic Interactions.” OSF Preprints. February 27. doi:10.31219/osf.io/y5v6d. 